\title{
Many-body solitons in a one-dimensional condensate of hard core bosons
}

\author{
M. D. Girardeau ${ }^{1,2}$ and E. M. Wright ${ }^{2}$ \\ ${ }^{1}$ Institute of Theoretical Science, University of Oregon, Eugene, OR 97403 \\ ${ }^{2}$ Optical Sciences Center and Department of Physics, University of Arizona, Tucson, AZ 85721
}

(November 13, 2018)

\begin{abstract}
A mapping theorem leading to exact many-body dynamics of impenetrable bosons in one dimension reveals dark and gray soliton-like structures in a toroidal trap which is phaseimprinted. On long time scales revivals appear that are beyond the usual mean-field theory.
\end{abstract}

03.75.Fi,03.75.-b,05.30.Jp

Dark and gray solitons are a generic feature of the nonlinear Schrödinger equation with repulsive interactions, and several calculations of their dynamics based on the Gross-Pitaevskii (GP) equation have appeared [1] 8), as well as experiments demonstrating their existence in atomic BECs [5,6]. Since the GP equation is a nonlinear approximation to the more exact linear many-body Schrödinger equation, this raises the question of how observed solitonic behavior arises in a theory which is linear at a fundamental level. Here this issue will be examined with the aid of exact many-body solutions. It has been shown by Olshanii [9] that at sufficiently low temperatures, densities, and large positive scattering length, a BEC in a thin atom waveguide has dynamics which approach those of a one-dimensional (1D) gas of impenetrable point bosons. This is a model for which the exact many-body energy eigensolutions were found in 1960 using an exact mapping from the Hilbert space of energy eigenstates of an ideal gas of fictitious spinless fermions to that of many-body eigenstates of impenetrable, and therefore strongly interacting, bosons 110,11]. The term "Bose-Einstein condensation" is used here in a generalized sense; it was shown by Lenard [12] and by Yang and Yang 13] that for the many-boson ground state of this system, the occupation of the lowest single-particle state is of order $\sqrt{N}$ where $N$ is the total number of atoms, in contrast to $N$ for usual BEC. Nevertheless, since $N \gg 1$ and the momentum distribution has a sharp peak in the neighborhood of zero momentum [9], this system shows strong coherence effects typical of BEC. The response of a BEC of this type to application of a delta-pulsed optical lattice was recently calculated by Rojo et al. [14], using the Fermi-Bose mapping theorem [10,11], as an exactly calculable model of dynamical optical lattice behavior. They found spatial focussing and periodic self-imaging (Talbot effect), which decay as a result of interactions. This decay is absent in the GP approximation and therefore serves as a signature of many-body interaction effects omitted in GP.
In this Letter we examine the appearence of dark soliton-like structures using the model of a 1D hard-core Bose gas in a toroidal trap, or ring, with cross section so small that motion is essentially circumferencial [15, 19]. The Fermi-Bose mapping is employed to generate exact solutions for this problem. We identify stationary solutions which reflect some properties of dark solitons from the GP theory when the ring is pierced at a point by an intense blue-detuned laser. We also present dynamical solutions when half of an initially homogeneous ring BEC is phase-imprinted via the light-shift potential of an applied laser, leading to gray soliton-like structures whose velocity depends on the imposed phase-shift [5]6]. Such structures are apparent for times less than the echo time $\tau_{e}=L / c$, with $L$ the ring circumference and $c$ the speed of sound in the BEC. On longer time scales the dynamics becomes very complex showing Talbot recurrences which are beyond the GP theory.

Time-dependent Fermi-Bose mapping theorem: The original proof 10.11] was restricted to energy eigenstates, but the generalization to the time-dependent case is almost trivial. The Schrödinger Hamiltonian is assumed to have the structure

$$
\hat{H}=\sum_{j=1}^{N}-\frac{\hbar^{2}}{2 m} \frac{\partial^{2}}{\partial x_{j}^{2}}+V\left(x_{1}, \cdots, x_{N} ; t\right),
$$

where $x_{j}$ is the one-dimensional position of the $j$ th particle and $V$ is symmetric (invariant) under permutations of the particles. The two-particle interaction potential is assumed to contain a hard core of 1D diameter $a$. This is conveniently treated as a constraint on allowed wave functions $\psi\left(x_{1}, \cdots, x_{N} ; t\right)$ :

$$
\psi=0 \quad \text { if } \quad\left|x_{j}-x_{k}\right|<a \quad, \quad 1 \leq j<k \leq N,
$$

rather than as an infinite contribution to $V$, which then consists of all other (finite) interactions and external potentials. The time-dependent version starts from fermionic solutions $\psi_{F}\left(x_{1}, \cdots, x_{N} ; t\right)$ of the timedependent many-body Schrödinger equation (TDMBSE) $\hat{H} \psi=i \hbar \partial \psi / \partial t$ which are antisymmetric under all particle pair exchanges $x_{j} \leftrightarrow x_{k}$, hence all permutations. As in the original theorem [10], define a "unit antisymmetric function"

$$
A\left(x_{1}, \cdots, x_{N}\right)=\prod_{1 \leq j<k \leq N} \operatorname{sgn}\left(x_{k}-x_{j}\right)
$$


where $\operatorname{sgn}(x)$ is the algebraic sign of the coordinate difference $x=x_{k}-x_{j}$, i.e., it is $+1(-1)$ if $x>0(x<0)$. For given antisymmetric $\psi_{F}$, define a bosonic wave function $\psi_{B}$ by

$$
\psi_{B}\left(x_{1}, \cdots, x_{N} ; t\right)=A\left(x_{1}, \cdots, x_{N}\right) \psi_{F}\left(x_{1}, \cdots, x_{N} ; t\right)
$$

which defines the Fermi-Bose mapping. $\psi_{B}$ satisfies the hard core constraint (2) if $\psi_{F}$ does, is totally symmetric (bosonic) under permutations, obeys the same boundary conditions as $\psi_{F}$, e.g. periodic boundary conditions on a ring, and $\hat{H} \psi_{B}=i \hbar \partial \psi_{B} / \partial t$ follows from $\hat{H} \psi_{F}=i \hbar \partial \psi_{F} / \partial t$ 10,11.

Exact solutions for impenetrable point bosons: The mapping theorem leads to explicit expressions for all many-body energy eigenstates and eigenvalues of a 1D scalar condensate (bosons all of the same spin) under the assumption that the only two-particle interaction is a zero-range hard core repulsion, represented by the $a \rightarrow 0$ limit of the hard-core constraint. Such solutions were obtained in Sec. 3 of the original work 10] for periodic boundary conditions and no external potential. The exact many body ground state was found to be a pair product of Bijl-Jastrow form: $\psi_{0}=$ const. $\prod_{i>j}\left|\sin \left[\pi L^{-1}\left(x_{i}-x_{j}\right)\right]\right|$. In spite of the very long range of the individual pair correlation factors $\left|\sin \left[\pi L^{-1}\left(x_{i}-x_{j}\right)\right]\right|$, the pair distribution function $D\left(x_{i j}\right)$, the integral of $\left|\psi_{0}\right|^{2}$ over all but two coordinates, was found to be of short range: $D\left(x_{i j}\right)=1-j_{0}^{2}\left(\pi \rho x_{i j}\right)$, with $j_{0}(\xi)=\sin \xi / \xi$, the spherical Bessel function of order zero. The system was found to support propagation of sound with speed $c=\pi \hbar \rho / m$ where $\rho=N / L$, the 1D atom number density.

To generalize to the time-dependent case, assuming that the many-body potential of Eq. (1) is a sum of one-body external potentials $V\left(x_{j}, t\right)$, one generalizes the time-independent determinantal many-fermion wavefunction [10] to a determinant

$$
\psi_{F}\left(x_{1}, \cdots, x_{N} ; t\right)=C \underset{i, j=1}{N} \phi_{i}\left(x_{j}, t\right),
$$

of solutions $\phi_{i}(x, t)$ of the one-body TDSE in the external potential $V(x, t)$. It then follows that $\psi_{F}$ satisfies the TDMBSE, and it satisfies the impenetrability constraint (vanishing when any $x_{j}=x_{\ell}$ ) trivially due to antisymmetry. Then by the mapping theorem $\psi_{B}$ of Eq.(4) satisfies the same TDMBSE.

Dark solitons on a ring: Consider $N$ bosons in a tight toroidal trap, and denote their 1D positions measured around the circumference by $x_{j}$. This is equivalent to the exactly-solved model [10] of $N$ impenetrable point bosons in $1 \mathrm{D}$ with wave functions satisfying periodic boundary conditions with period $L$ equal to the torus circumference, and the fundamental periodicity cell may be chosen as $-L / 2<x_{j}<L / 2$. However, the rotationally invariant quantum states of this problem do not reveal any dark soliton-like structures. To proceed we therefore consider the case that a blue-detuned laser field pierces the ring at $x=0$ by virtue of the associated repulsive dipole force: The light sheet then provides a reference position for the null of the dark soliton. Assume that the light sheet is so intense and narrow that it may be replaced by a constraint that the many-body wave function (hence the orbitals $\phi_{i}$ ) must vanish whenever any $x_{j}=0$. Then the appropriate orbitals $\phi_{i}(x)$ are free-particle energy eigenstates vanishing at $x=0$ and periodic with period $L$. The complete orthonormal set of even-parity eigenstates $\phi_{n}^{(+)}$and odd-parity eigenstates $\phi_{n}^{(-)}$are

$$
\begin{aligned}
& \phi_{n}^{(+)}(x)=\sqrt{2 / L} \sin [(2 n-1) \pi|x| / L], \\
& \phi_{n}^{(-)}(x)=\sqrt{2 / L} \sin (2 n \pi x / L),
\end{aligned}
$$

with $n$ running from 1 to $\infty$. The odd eigenstates are the same as those of free particles with no $x=0$ constraint, since these already vanish at $x=0$. However, the even ones are strongly affected by the constraint, their cusp at $x=0$ being a result of the impenetrable light sheet at that point. If one bends a $1 \mathrm{D}$ box $-L / 2<x<L / 2$ with impenetrable walls into a ring, identifying the walls at $\pm L / 2$, then those particle-in-a-box eigenfunctions which are even about the box center become identical with the $\phi_{n}^{(+)}$, and their cusp results from the nonzero slope of these functions at the walls. The $N$-fermion ground state is obtained by inserting the lowest $N$ orbitals (6) into the determinant (5) (filled Fermi sea). Assume that $N$ is odd. Since $\phi_{1}^{(+)}$is lower than $\phi_{1}^{(-)}$, this Fermi sea consists of the first $(N+1) / 2$ of the $\phi_{n}^{(+)}$and the first $(N-1) / 2$ of the $\phi_{n}^{(-)}$. The $N$-boson ground state is then given by (4). Since $A^{2}=1$, its one-particle density $\rho(x)$ is the same as that of the $N$-fermion ground state, the sum of partial densities contributed by all one-particle states in the Fermi sea. Thus it is the sum of

$$
\rho^{(+)}(x)=\frac{N+1}{2 L}-\frac{\sin [2(N+1) \pi x / L]}{2 L \sin (2 \pi x / L)},
$$

and

$\rho^{(-)}(x)=\frac{N-1}{2 L}-\frac{\sin [(N-1) \pi x / L] \cos [(N-3) \pi x / L]}{L \sin (2 \pi x / L)}$

In the thermodynamic limit $N \rightarrow \infty, L \rightarrow \infty, N / L \rightarrow \rho$ for fixed $x, \rho^{( \pm)}$each contribute half of the total density $\rho(x)$ :

$$
\rho(x) \sim \rho\left[1-j_{0}(2 \pi \rho x)\right] .
$$

Since $j_{0}(0)=1, \rho(x)$ vanishes at $x=0$ and approaches the mean density $\rho$ over a healing length $L_{h}=1 / 2 \rho$ with damped spatial oscillations about its limiting value. This differs in detail from the density $\rho_{\infty} \tanh ^{2}(x / w)$ of a GP dark soliton [20], with $\rho_{\infty}$ the background density and 
$w$ the corresponding healing length, but has some qualitative similarity. However, it is only the odd component $\rho^{(-)}(x) \approx \rho(x) / 2$ which has the feature of a dark soliton that the corresponding odd orbitals have a $\pi$ phasejump at $x=0$ (and also at $x= \pm L / 2$ to obey the periodic boundary conditions). But the odd and even components can never be separated physically, so the odd dark soliton-like component is always accompanied by the even non-soliton component.

Next, suppose that the light-sheet is turned off at $t=0$ by removing the constraint that the wave function vanish at $x=0$. The solution of the TDMBSB for the many-boson system is then given by (4) where the Slater determinant (5) is built from the first $(N+1) / 2$ of the $\phi_{n}^{(+)}(x, t)$ and the first $(N-1) / 2$ of the $\phi_{n}^{(-)}(x, t)$, where these time-dependent orbitals are solutions of the singlefree-particle TDSE which (a) reduce to the orbitals (6) at $t=0$, and (b) satisfy periodic boundary conditions with periodicity cell $-L / 2<x<L / 2$. The odd solutions are trivial: Since these never "see" the $x=0$ constraint even for $t<0$, they differ from the odd orbitals (6) only by time-dependent phase shifts: $\phi_{n}^{(-)}(x, t)=\phi_{n}^{(-)}(x) e^{-i \omega_{n} t}$ with $\omega_{n}=\hbar k_{n}^{2} / 2 m$ and $k_{n}=2 n \pi / L$. It follows that $\rho^{(-)}(x, t)$ is time-independent, and given in the thermodynamic limit by

$$
\rho^{(-)}(x, t) \sim(\rho / 2)\left[1-j_{0}(2 \pi \rho x)\right] .
$$

This further reinforces our view that the odd component of the density shares features of a dark soliton. The even-parity orbitals $\phi_{n}^{(+)}(x, t)$ are complicated since the removal of the light sheet constitutes a large, sudden perturbation. Indeed, the periodic even-parity solutions of the free-particle Schrödinger equation are $\chi_{p}^{(+)}(x)=$ $\sqrt{\left(2-\delta_{p 0}\right) / L} \cos (2 p \pi x / L)$ with $p=0,1,2, \cdots$, and these are very different from the solutions $\phi_{n}^{(+)}(x)$ with the $x=0$ constraint [Eq. (6)]. Nevertheless, since the $\chi_{p}^{(+)}(x)$ are complete for the subspace of even-parity, spatially periodic functions, one can expand the $\phi_{n}^{(+)}(x, t)$ in terms of the $\chi_{p}^{(+)}(x)$, which evolve with time-dependent phases $e^{-i \omega_{p} t}$ with $\omega_{p}=\hbar k_{p}^{2} / 2 m$ and $k_{p}=2 p \pi / L$. One finds

$$
\phi_{n}^{(+)}(x, t)=\frac{2(2 n-1)}{\pi} \sqrt{\frac{2}{L}} \sum_{p=0}^{\infty} \frac{\left(2-\delta_{p 0}\right) \cos \left(k_{p} x\right) e^{-i \omega_{p} t}}{(2 n-1)^{2}-4 p^{2}}
$$

$\rho^{(+)}(x, t)$ is the sum of absolute squares of the first $(N+1) / 2$ of the sums (11), generalizing (7). Adding the time-independent expression $\rho^{(-)}(x, t)$, given in the thermodynamic limit by (10) or exactly by (8), one finds the time-dependent total density $\rho(x, t)$. There are two important time scales: One is the Poincaré recurrence time $\tau_{r}$. Noting that $\omega_{p}$ in (11) is proportional to $p^{2}$, one finds that all terms in the sum are time-periodic with pe$\operatorname{riod} \tau_{r}=m L^{2} / \pi \hbar$, which is therefore the recurrence time for the density and in fact all properties of our model [14]. The other important time is the echo time $\tau_{e}$, the time for sound to make one circuit around the torus. Recalling that the speed of sound in this system is $c=\pi \hbar \rho / m$ [10], one finds $\tau_{e}=\tau_{r} / N$. For $t<<\tau_{e}$ after the constraint is removed, the initial density develops sound waves that propagate around the ring, and that we examine below in the context of phase-imprinting. For $t>\tau_{e}$ the evolution is very complex, but complete recurrences occur for times $t=n \tau_{r}$ with fractional revivals in between.

Gray soliton formation by phase-imprinting: Consider next a toroidal BEC in its ground state to which a phaseimprinting laser is applied over half the ring at $t=0$. This is described by the single-particle Hamiltonian

$$
\hat{H}=\sum_{j=1}^{N}\left[-\frac{\hbar^{2}}{2 m} \frac{\partial^{2}}{\partial x_{j}^{2}}-\hbar \Delta \theta \delta(t) S\left(x_{j}\right)\right]
$$

where $S(x)=\theta(L / 4-|x|)$, i.e., unity for $-L / 4<x<$ $L / 4$ and zero elsewhere. This is the technique used in recent experiments [5.,6], here idealized to a delta-function in time and to sharp spatial edges. Before the pulse the most convenient free-particle orbitals in (5) are plane waves $\phi_{n}(x)=\sqrt{(1 / L)} e^{i k_{n} x}$ where $k_{n}=2 n \pi / L$ and $n=-n_{F},-n_{F}+1, \cdots, n_{F}-1, n_{F}$ with $n_{F}=(N-1) / 2$. Let $\phi_{n}(x, t)$ be the solution of the TDSE with the Hamiltonian (12) reducing to the above $\phi_{n}(x)$ just before the pulse. Then the solutions just after the pulse are $\phi_{n}(x, 0+)=\phi_{n}(x) e^{i S(x) \Delta \theta}$. The potential gradients at the pulse edges impart momentum kicks to the particles there which induce both compressional waves propagating at the speed, $c$, of sound and density dips (gray solitons) moving at speeds $|v|<c$. The expansion of $\phi_{n}(x, t)$ in terms of the unperturbed plane waves is evaluated as

$$
\begin{aligned}
\phi_{n}(x, t) & =\frac{1}{2}\left(1+e^{i \Delta \theta}\right)-\frac{1-e^{i \Delta \theta}}{\pi} \sum_{\ell=-\infty}^{\infty} \\
& \times \frac{(-1)^{\ell} \phi_{n-2 \ell-1}(x) e^{-i \omega_{n-2 \ell-1} t}}{2 \ell+1}
\end{aligned}
$$

and the time-dependent density is the sum of the absolute squares of the lowest $N$ of these. Figure 1 shows numerical simulations obtained using Eq. (13) for $N=51$, $t / \tau_{e}=0.051$, and $\Delta \theta=\pi$ (solid line), and $\Delta \theta=0.5 \pi$ (dashed line): due to symmetry we show only half of the ring $-L / 2<x<0$, the phase-shift being imposed at $x=-L / 4$. Considering times short compared to the echo time means that the corresponding results are not very sensitive to the periodic boundary conditions, and also therefore apply to a linear geometry. The initial density profile is flat with a value $\rho_{0} L=51$. For both phase-shifts two distinct maxima are seen, which travel at close to the speed of sound $c$, and two distinct min- 
ima, which are analogous to gray solitons and travel at velocities $|v| / c<1$.

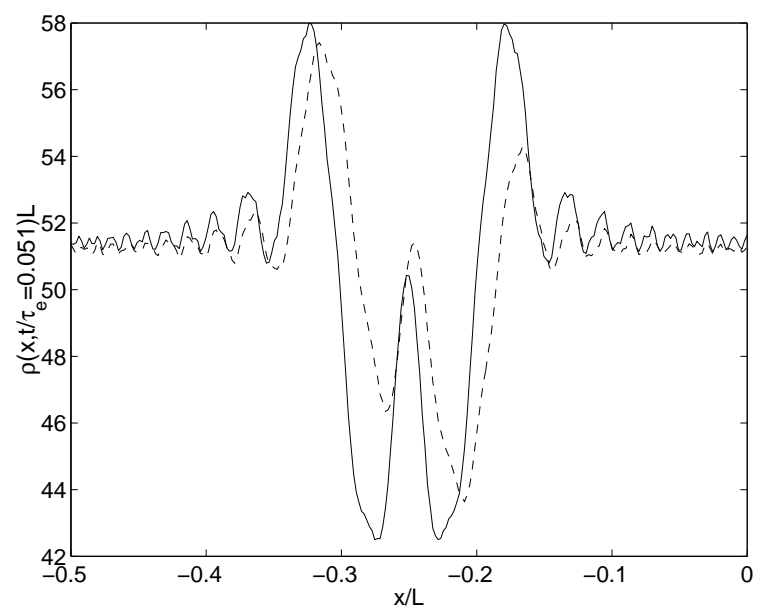

FIG. 1. Scaled density $\rho(x, t) L$ versus scaled position around the ring $x / L$ for $N=51, t / \tau_{e}=0.051$, and $\Delta \theta=\pi$ (solid line), and $\Delta \theta=0.5 \pi$ (dashed line). Due to symmetry we show only half of the ring $-L / 2<x<0$, the phase-jump being imposed at $x=-L / 4$.

In addition, there are also high wavevector oscillations which radiate at velocities greater than $c$. In the case of a phase-shift $\Delta \theta=\pi$, the density is symmetric about $x=$ $-L / 4$, whereas for a phase-shift other than a multiple of $\pi$ the evolution is not symmetric, see the dashed line where the global minimum moves to the right in reponse to the phase-shift.

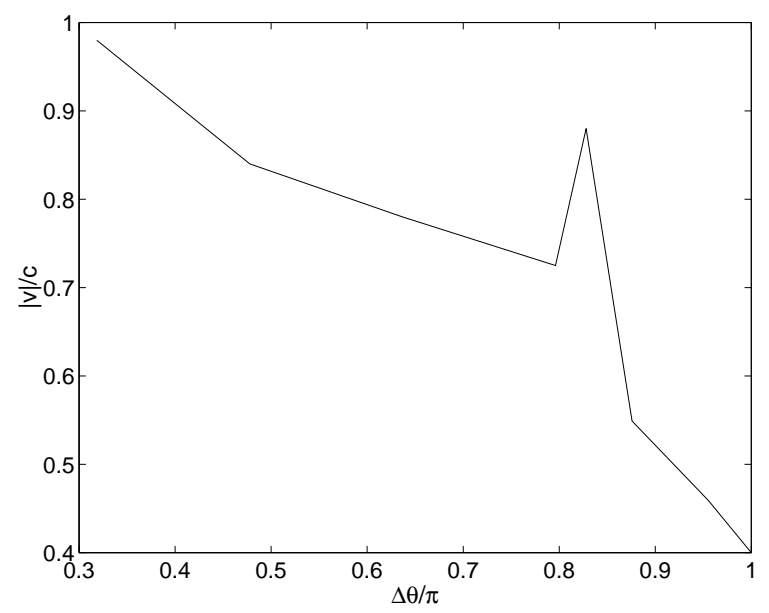

FIG. 2. Dark soliton velocity $|v| / c$ scaled to the speed of sound $c$ as a function of phase-shift $\Delta \theta / \pi$ for $N=51$.

In Fig. 2 we plot the calculated velocity of the global density minimum relative to the speed of sound for a variety of phase-shifts $\Delta \theta$. The basic trend is that larger phase-shift means lower velocity, in qualitative agreement with recent experiments [5.6], but there is a sharp velocity peak at $\Delta \theta \approx 0.83 \pi$ : This peak results from the crossover between two local minima in the density. These gen- eral features, the generation of gray solitons and density waves, agree with those of the GP theory, but here arise out of the exact many-body calculation.

In conclusion, using our exactly-soluble 1D model we hope to have shown that the dark solitonic features of atomic BECs normally described within the mean-field GP theory arise naturally from consideration of the exact linear many-body theory for times less than the echo time. An advantage of this approach is that it is numberconserving and does not rely on any symmetry-breaking approximation. In addition, long time dynamics such as collapses and revivals are accounted for 14]. A detailed comparison between our results and current experiments is not possible as they do not conform to the conditions for a $1 \mathrm{D}$ system. However, some estimates are in order to set the appropriate time scales: If we consider ${ }^{87} \mathrm{Rb}$ with a ring of circumference $L=100 \mu \mathrm{m}$, and a high transverse trapping frequency $\omega_{\perp}=2 \pi \times 10^{5} \mathrm{~Hz}$, then we are limited to atom numbers $N<300$ [9], so these are small condensates. We then obtain $\tau_{r}=4.6$ s, and $\tau_{e}=90 \mathrm{~ms}$ for $N=51$. Finally, we remark that since our approach relied on the mapping between the strongly-interacting Bose system and a non-interacting "spinless Fermi gas" model, this suggests that dark and gray solitons should also manifest themselves in the density for the 1D Fermi system. Although real fermions have spin, the interactions used here to generate solitons were spin-independent.

This work was supported by the Office of Naval Research Contract No. N00014-99-1-0806.

[1] W.P. Reinhardt and C.W. Clark, J. Phys. B 30, L785 (1997).

[2] R. Dum et al., Phys. Rev. Lett. 80, 2972 (1998).

[3] T.F. Scott, R.J. Ballagh, and K. Burnett, J. Phys. B 31, L329 (1998).

[4] A.D. Jackson, G.M. Kavoulakis, and C.J. Pethick, Phys. Rev. A 58, 2417 (1998).

[5] S. Burger et al., Phys. Rev. Lett. 83, 5198 (1999).

[6] J. Denshlag et al. Science 287, 97 (1999).

[7] A.E. Muryshev et al., Phys. Rev. A 60, R2665 (1999).

[8] Th. Busch and J.R. Anglin, cond-mat/9809408.

[9] M. Olshanii, Phys. Rev. Lett. 81, 938 (1998).

[10] M. Girardeau, J. Math. Phys. 1, 516 (1960).

[11] M.D. Girardeau, Phys. Rev. 139, B500 (1965). See particularly Secs. 2, 3, and 6 .

[12] A. Lenard, J. Math. Phys. 7, 1268 (1966).

[13] C.N. Yang and C.P. Yang, J. Math. Phys. 10, 1115 (1969).

[14] A. G. Rojo, G. L. Cohen, and P. R. Berman, Phys. Rev. A 60, 1482 (1999).

[15] J. Javanainen, S. M. Paik, S. M. Yoo, Phys. Rev. A 58, 
580 (1998).

[16] L. Salasnich, A. Parola, and L. Reatto, Phys. Rev. A 59, 2990 (1999).

[17] Th. Busch and J. R. Anglin, Phys. Rev. A 60, R2669 (1999).

[18] M. Benlaki et al., cond-mat/9711295.

[19] D. S. Rokhsar, cond-mat/9709212. Science 275, 637 (1997).

[20] V.L. Ginzburg and L.P. Pitaevskii, Soviet Phys. JETP 34, 858 (1958), Sec. 2; P.O. Fedichev, A.E. Muryshev, and G.V. Shlyapnikov, Phys. Rev. A 60, 3220 (1999). 